\title{
Cosmic Ray Science Potential for an Extended Surface array at the IceCube Observatory
}

The IceCube-Gen2 Collaboration ${ }^{1}$,

${ }^{1}$ http://icecube.wisc.edu/collaboration/authors/icrc15_gen2

E-mail: seckel@bartol.udel.edu

IceTop is the $1 \mathrm{~km}^{2}$ surface array of the IceCube Neutrino Observatory. Measurements of ground level particles by IceTop have been used for high resolution measurements of the cosmic ray spectrum for energies of 2-500 PeV. Composition has been studied by considering coincident measurements of $\mathrm{TeV}$ muon bundles in the $2 \mathrm{~km}$ deep IceCube neutrino detector. Including IceTop data for $\mathrm{GeV}$ muons may reduce uncertainties inherent to hadronic interaction models; however, all three parts of this program are somewhat limited by the size of IceTop. We explore the potential for IceCube-Gen2 with surface components (IceTop-2, "veto") to improve the cosmic ray science program. The increased area would permit a factor of $\sim 3$ increase in energy range for the spectral measurement, a factor of 50 increase in coincident data, and enable lateral and production depth muon measurements for every event.

Corresponding authors: David Seckel ${ }^{1 *}$,

${ }^{1}$ Department of Physics and Astonomy, University of Delaware, Newark DE 19716, USA

The 34th International Cosmic Ray Conference,

30 July- 6 August, 2015

The Hague, The Netherlands

${ }^{*}$ Speaker. 
IceCube [1], IceTop [2], and cosmic rays. Although best known for its contributions to neutrino physics, IceCube is also a significant cosmic ray observatory. IceCube has produced a high resolution cosmic ray energy spectrum [3,4] based on data purely from IceTop, the surface array directly above the main in-ice neutrino detector (Fig. 1). By including in-ice data characterizing the muon bundles associated with coincident air shower events, IceCube is providing a unique composition measurement $[3,5]$ between the knee and the ankle of the cosmic ray spectrum. We are studying the ability to separate the surface response into electromagnetic (em ) and muonic components $[6,7]$, which we will use to produce an independent proxy for the mass of the cosmic ray primary responsible for an air shower. These analyses feed into a community effort to understand cosmic rays in the range $10^{16}-10^{18} \mathrm{eV}$, where there is expected to be a transition from galactic to extragalactic origins [8]. All three efforts are somewhat limited by the $1 \mathrm{~km}^{2}$ extent of the IceTop array, and so the upper end of the transition is not well covered, especially with regards to composition.

At the same time, IceCube has completed one of its main science goals by demonstrating the existence of an astrophysical neutrino flux $[9,10]$. This result is one of the driving considerations for IceCube-Gen2 [11, 12], a second generation detector at South Pole. Gen2 is planned on the same principles as IceCube but with roughly an order of magnitude better sensitivity for achieving key science goals related to the high energy astrophysical neutrino flux. This increase derives primarily from larger area and an enhanced surface veto $[13,14]$ to increase the significance of astrophysical neutrino events relative to a background of air shower induced muons and neutrinos.

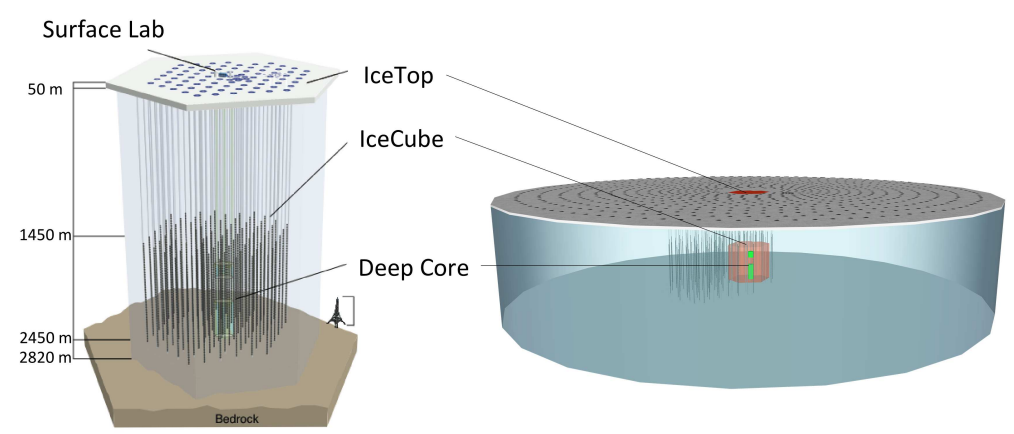

Figure 1: Left: IceCube neutrino observatory. Right: IceCube-Gen2 with extended version of surface veto.

The increase in area and a dedicated veto array provide an opportunity to improve existing analyses at the higher energies. A nominal plan is to deploy at each string a surface station capable of good timing and charge measurements with high dynamic range to provide a quality reconstruction of the size and geometry of the air shower event. As part of Gen2 this would permit extending both the surface only analysis and the coincident in-ice/surface analysis to above $1 \mathrm{EeV}$ in energy. The improvement in the coincident analysis is most significant, roughly a factor of 50 by simple geometry considerations. In addition, a veto array could permit an unprecedented measurement of the peripheral muons in an air shower, complementing the combined in-ice/surface measurement of cosmic ray composition.

IceCube cosmic ray science program. IceCube's cosmic ray program includes two distinct determinations of the all-particle cosmic ray energy spectrum, both based on 3 years of data. The first, 
utilizing just IceTop data, is shown in Fig. 2 along with data from other experiments [15] spanning the energy range from the knee to above the ankle. It is notable that the IceTop result is a relatively high resolution spectrum compared to other experiments at energies below $\sim 300 \mathrm{PeV}$, reflecting superior statistics. The gray band showing systematic uncertainty mostly affects the overall normalization (see [3, 4]). The IceTop result shows clearly that the region from the knee to the ankle cannot be described by a simple power law. At higher energies, there is some tension between Auger and TA, but present IceTop statistics are not sufficient to provide any insight. In fact, there are 18 events with energies above $10^{9.1} \mathrm{GeV}$ which are not shown due to an increase in systematic uncertainty as large showers saturate a significant fraction of the array, even on kilometer scales.

The IceTop only analysis assumes a specific energy dependent composition in deriving the spectrum, and that assumption is included in the systematic uncertainties. The second (coincident) analysis relaxes this assumption and uses in-ice data to infer composition. Muon bundles produced in the air shower event penetrate to the in-ice array. Since heavier nuclei produce more muons, the in-ice signal is a proxy for the mass of the cosmic ray primary. The analysis utilizes a neural net to produce a weight for each event to arise from one of four nuclear mass groups. By combining the weights from all particles, a composition is determined as a function of primary energy. The result is shown in Fig. 3. In each panel the all-particle spectrum is shown in black above the partial spectrum for a particular mass group. The all-particle energy spectrum is in good agreement with that derived from IceTop alone. The light species dominate at the knee, and composition gets heavier with increasing energy, but detailed interpretation is pending a fuller discussion of systematics, including calibration of the response to in-ice muon bundles and variations due to different hadronic interaction models [3]. The coincident analysis has a restricted energy range compared to the IceTop only analysis, with statistics limited by the coincident geometry which reduces the sky coverage from $\Omega_{t o p}=1.26 \mathrm{sr}$ to $\Omega_{c o} \sim 0.15 \mathrm{sr}$.

In principle, IceTop can also be used to measure the muon content of air showers [6, 7]; however, it is difficult to observe individual muons in the inner part of the air shower where the $\mathrm{em}$ part of the shower is intense. At energies above a few PeV, the inner $300 \mathrm{~m}$ is not available to muon counting. At higher energies, one must go further out, but a shower which passes the containment cuts is never more than $900 \mathrm{~m}$ from the furthest IceTop station. There may be few observable muons with which to build a lateral distribution function (LDF) and characterize the event.

The IceCube cosmic ray program includes several more specialized analyses which would benefit from IceCube-Gen2. a) Searching for $\gamma$-rays is an extreme form of composition study. Air showers with abnormally low muon content are $\gamma$-ray candidates. These may be found within the coincident geometry [16], or as events where the surface muon content is low. Selection criteria may be optimized to look for a diffuse flux, or a source defined by a cluster in sky position and/or time. b) Neutrons are another neutral component of the cosmic ray spectrum, relying on an excess of clustered events for detection [17]. c) Both IceTop and IceCube(in-ice) are used to study anisotropy in different ranges of energy and declination $[18,19,20]$.

Improvements with IceCube-Gen2. For the present discussion, it is convenient to adopt a baseline configuration for IceCube-Gen2 that includes an in-ice array and two surface components, which we will refer to as "the veto" and IceTop-2 (IT2). The in-ice array is nominally taken to be $7 \mathrm{~km}^{2}$ in area - middle of the range considered in [12]. The veto is designed to enhance Gen2 per- 


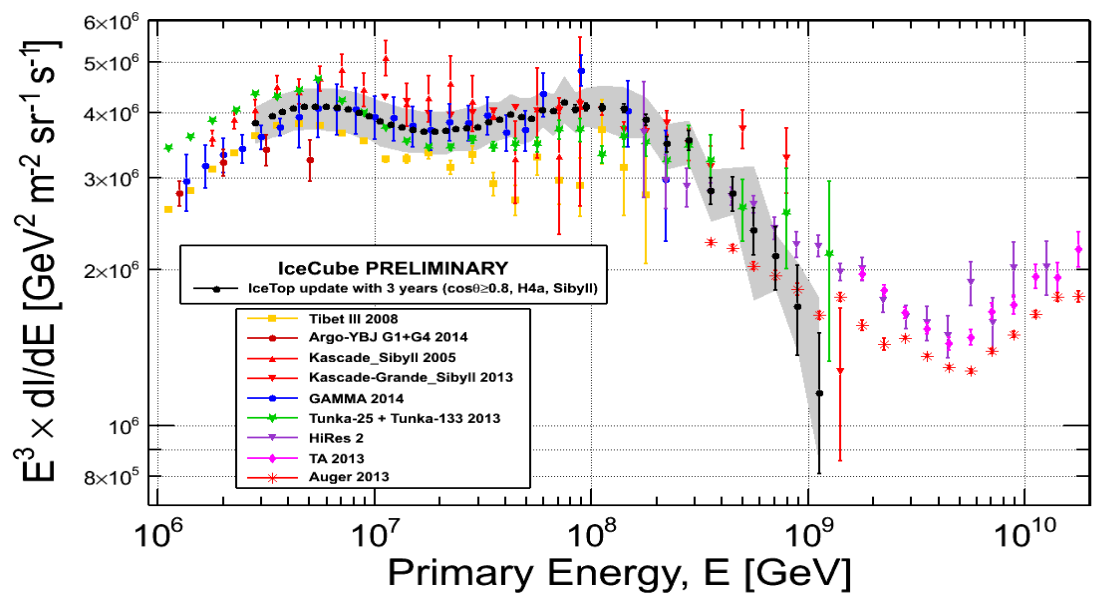

Figure 2: Cosmic ray all-particle energy spectrum using 3 years of IceTop data. Error bars show statistics, gray band shows systematic uncertainty.
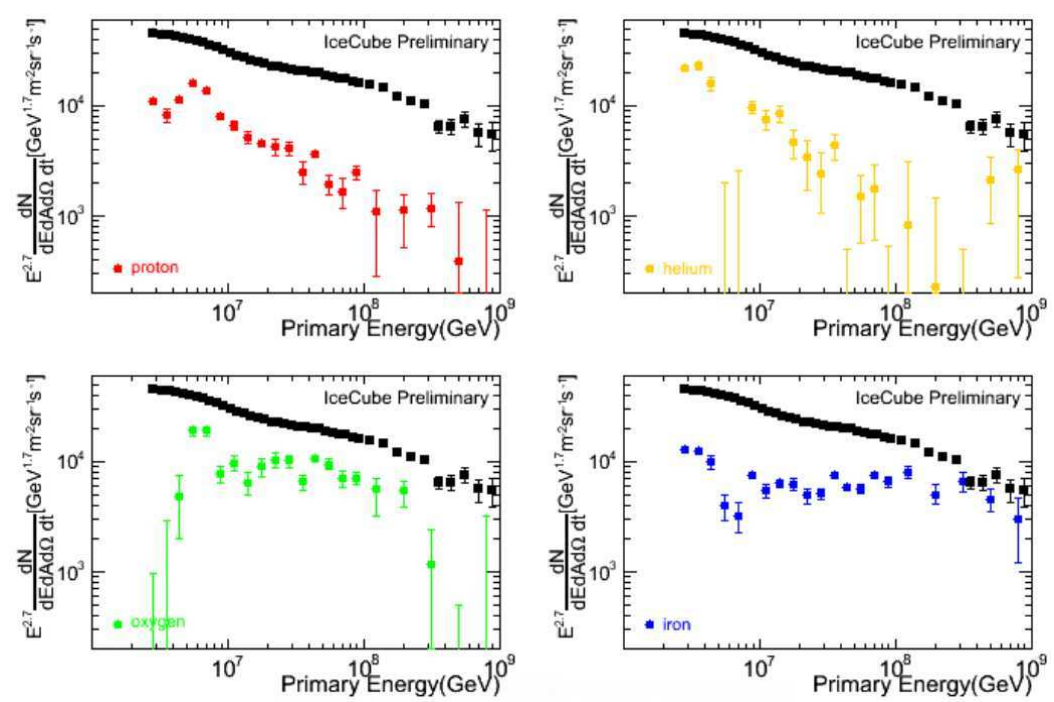

Figure 3: Coincident 3 year spectrum by mass, with all-particles in black. (statistical uncertainties only)

formance with respect to high energy neutrino science. Here, the baseline veto design is taken to be a dense array of particle detectors deployed over the in-ice footprint, although other approaches may be viable [13]. Reasons for this choice are summarized below. Cost efficiency and design goals for the veto may affect its value for measuring properties of extensive air showers. Accordingly, IceTop-2 would sample the ground level footprint of an air shower with timing and charge resolution sufficient to enable good reconstruction of the size and geometry of the air shower. The baseline for IT2 is to place a station at the head of each string, but we do not specify the instrumentation other than to assume that it can produce reconstructions comparable to those produced by the current IceTop, just at higher energies commensurate with larger spacing between stations.

Improvements due to IceTop-2. The improvements are primarily a consequence of increased 
size. For current IceTop, the surface only analysis includes a fiducial area of $A=0.62 \mathrm{~km}^{2}$, somewhat smaller than the actual instrumented area of $0.83 \mathrm{~km}^{2}$, driven largely by the need to reconstruct the core location of the air shower. For the Gen2 estimates we will use a fiducial area of $6 \mathrm{~km}^{2}$, noting that we expect the edge effects to be reduced and that including data from the veto will improve efficiency. For the surface only analysis, we anticipate an order of magnitude better statistics. Referring to Fig. 2, we expect the scatter of points around $300 \mathrm{PeV}$ to either be smoothed, or to resolve into clear features. Above an EeV, 100 events/yr $(10 \times$ IceTop $)$ will accumulate to provide a meaningful overlap with Auger and TA.

The improvements for the coincident analysis are more dramatic. For estimation, we assume the same containment cut for both surface and in-ice arrays. For IceCube, the coincident solid angle is $\Omega_{c o} \simeq A / d^{2}=0.15 \mathrm{sr}$, where $d=2 \mathrm{~km}$ is the depth to the center of the in-ice array. This yields an aperture of $\langle A \Omega\rangle=0.09 \mathrm{~km}^{2}$ sr, which can be compared to $\langle A \Omega\rangle=0.70 \mathrm{~km}^{2}$ sr for the surface only analysis, and a ratio of events surviving all cuts of about $N_{\text {top }}: N_{c o}:: 8: 1$. For Gen2, the aperture for two disks with $A=6 \mathrm{~km}^{2}$, separated by $2 \mathrm{~km}$, is $\langle A \Omega\rangle=4.9 \mathrm{~km}^{2} \mathrm{sr}$, conservatively $\sim 50$ times that for current IceCube. It seems likely that galactic and extragalactic sources will have different compositions, either due to intrinsic injection or differential propagation, so increased coincident data informing on the changing composition will be of very high value. Other analyses which rely on coincident data (e.g. $\gamma$-ray search) will also benefit from increased sky coverage, including the eastern portion of the galactic plane.

In addition to the overall aperture and statistics, the increase in area from IceTop to IT2 should also improve the efficiency of analysis. At the upper end of IceTop's energy range the electromagnetic footprint saturates large fractions of the detector, increasing the systematic uncertainty and decreasing the effective area. The em blanket also obscures muons, making a two component analysis difficult at the highest energies. These effects will be mitigated by the larger area of IT2.

Planning a surface veto for Gen2. The fundamental challenge for IceCube and IceCube-Gen2 is to detect astrophysical neutrinos against a background of muons and neutrinos produced as a result of cosmic ray interactions in the atmosphere. Upward atmospheric muons are shielded by the Earth, but upward atmospheric neutrinos are an irreducible background for astrophysical neutrinos from the northern sky. The downward case is challenging since the flux of downward atmospheric muons must be eliminated, even at energies of order $100 \mathrm{TeV}$ [13]. However, if all cosmic ray events can be tagged, there is potential to be free not just of muons, but also atmospheric neutrinos. Further, the ice above the in-ice array may be used as a target volume, increasing the rate of track-like events, which have extra value in searches for point sources of astrophysical neutrinos [13].

We consider two veto strategies built around detecting ground level particles. The first focuses on sky coverage, seeking to cover a large fraction of the galaxy including the galactic center. This implies extending the veto to some $6 \mathrm{~km}$, with overall area of $100 \mathrm{~km}^{2}$. Assuming a minimal tagging threshold of a few $\mathrm{PeV}$, such a veto would detect cosmic rays with energies above $10 \mathrm{PeV}$. If detections were of sufficient quality all the arguments of the previous paragraphs would apply. A spectrum built only from surface observations would gain by another order of magnitude. Since the in-ice array would remain fixed, the coincident analysis would have a modest gain due to an increase in solid angle as the field of view was extended from zenith angle of 35 deg to $60 \mathrm{deg}$.

The alternative is to deploy a very dense array over the footprint of Gen2, capable of tagging 
cosmic rays with a passing rate of $10^{-4}-10^{-6}$ [21]. Preliminary estimates are that this could be achieved by an array with a filling factor of $10^{-3}$, e.g. roughly $7,000 \mathrm{~m}^{2}$ of scintillator paddles deployed over the baseline $7 \mathrm{~km}^{2}$. (For comparison, AMIGA (the Auger in-fill) employs $2000 \mathrm{~m}^{2}$ of shielded muon counters to provide a filling factor of $\sim 10^{-4}$ [22].) Although sensitive to em secondaries, the Gen2 design would be focused on muon detection to ensure veto capability at high inclinations, and to provide stable operation in the presence of snow accumulation on decadal time scales. This alternative is intriguing for cosmic ray science, as it presents the possibility of collecting hundreds of "peripheral" muons, contributing to the composition studies by Gen2.

Contribution of veto array to studies of cosmic ray composition. A counter for muons within an extensive air shower must isolate the muon signal from the electromagnetic part of the shower, either by shielding the muon counter, or by separating the muons from the em part of the shower in space and/or time. To this end, we define two interior regions for the muon LDF: a core with lateral separation $r_{\perp}<320 \mathrm{~m}$ common to all showers and an "umbra" where the muons are "shadowed" by em signals. The core sets the spacing of the veto array, but we define the peripheral muons to be those outside the umbra. The radius of the umbra depends on details of detector design, as well as energy and inclination of the air showers. If the veto elements include shielding, the umbra would have small radius allowing more muons to be counted. If the veto is sensitive to low energy electrons and $\gamma_{s}$, then the radius of the umbra is increased and surface muon yield decreases. In [6] the muon LDF is studied for IceTop, establishing that the umbra for quasi-vertical showers and IceTop tanks increases from $250 \mathrm{~m}$ to $600 \mathrm{~m}$ as primary energy increases from 3 to $30 \mathrm{PeV}$. We are studying the degree of shielding required for a scintillator array to maintain an umbra at $600 \mathrm{~m}$, so that $\sim \frac{1}{3}$ the muons can be used to study the hadronic nature of each air shower.

Fig. 4 shows the potential to use these peripheral muons to supplement composition studies, assuming that size and geometry of the air shower are reconstructed in IT2. In the left panel, the muon production is shown for a sample of simulated $\mathrm{p}$ and Fe showers. The simplest proxy for separating $\mathrm{p}$ and $\mathrm{Fe}$ is to count the total number of peripheral muons, multiply by the fractional coverage and compute an expected number of counts. At $30 \mathrm{PeV}$ this corresponds to $\mathrm{p} / \mathrm{Fe} \simeq 50 / 80$. Even with fluctuations this provides reasonable separation. In the right panel, a set of 1000 pseudoobservations of $30 \mathrm{PeV}$ events is shown. For each event, shower size is smeared by $15 \%$, setting IT2 resolution to that achieved by IceTop [4]. The expected number of muons with $r_{\perp}>600 \mathrm{~m}$ is then sampled to yield a simulated count which is shown on the $y$-axis. The $x$-axis shows a comparable quantity for the in-ice bundle. We count all muons with energy $E_{\mu}>340 / \cos \theta \mathrm{GeV}$, and then scatter that with a width of $\Delta \log _{10}\left[N_{\mu}\right]=0.1$, simulating the performance of IceCube bundle resolution [23]. At $30 \mathrm{PeV}$, the potential for the surface veto to separate $\mathrm{p} / \mathrm{Fe}$ is comparable to that already demonstrated for in-ice muons. By using both measures, $\mathrm{p} / \mathrm{Fe}$ separation may be improved and the main uncertainties of the combined analysis (in-ice calibration and hadronic interaction models) may be addressed.

The left panel of Fig. 4 also suggests that muon production depth [24] may be used to inform on nucleon mass. The solid histograms show the true production depth as reported by CORSIKA. The unfilled histograms (labeled "Reco") show a simple estimate of depth derived from a proxy for the emission height $h=r_{\perp}^{2} /(2 c \delta t)$ and an atmospheric density profile. Here $\delta t$ is the arrival delay of the muon relative to a planar shower front. Realizing a good muon production profile 

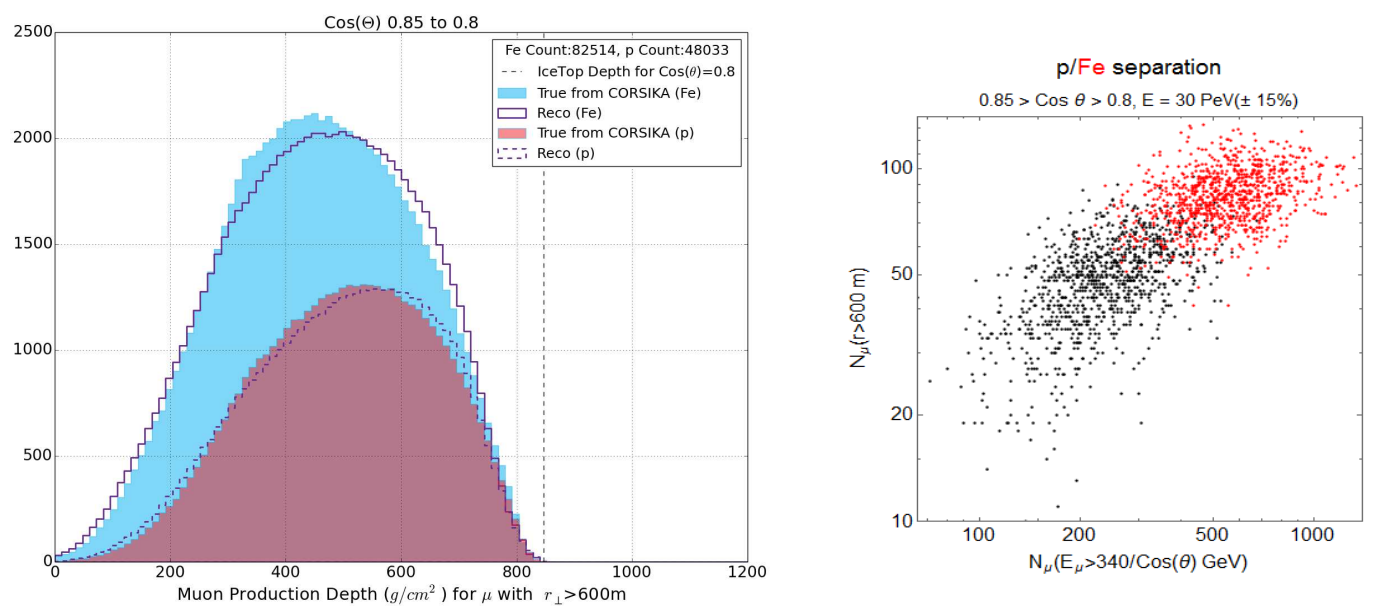

Figure 4: Potential for veto to contribute to composition studies (see text). Left: Average muon counts v $X_{\mu}$, for $25 \mathrm{p} / \mathrm{Fe}$ showers at $30 \mathrm{PeV}$. Right: $\mathrm{p} / \mathrm{Fe}$ separation showing both in-ice $(x)$ and surface muons $(y)$.

places requirements on a) the quality of IT2 reconstructed core positions, and b) the timing of muon signals from individual elements of the veto. Not only are earlier muons separated from the main part of the electromagnetic shower, but depth of muon production $\left(X_{\max , \mu},\left\langle X_{\mu}\right\rangle\right)$ provides some separation between $\mathrm{p}$ and Fe. The very early part of the shower $\left(X<200 \mathrm{~g} / \mathrm{cm}^{2}\right)$ is enriched for $\mathrm{Fe}$ showers relative to proton, and provides comparable separation to just using the bulk number of peripheral muons. Ultimately, with of order 100 muons to work with, we expect to fit the muon production depth to describe both the number of muons and the rise time of the muon signal, which will independently contribute to a mass resolution on a shower by shower basis.

Summary. IceCube executes a cosmic ray physics program with precision measurements of spectrum from 2-500 $\mathrm{PeV}$ and preliminary measurements of composition from 3-300 $\mathrm{PeV}$. The program is limited by the size of IceTop, especially with regard to in-ice and/or surface muon measurements which are critical for measuring composition. IceCube-Gen2 will present a 5-10 times larger footprint and $\sim 50 \mathrm{x}$ larger coincident aperture. Two surface components are considered. IceTop-2 would enable continuing the cosmic ray program with the larger aperture, significantly improving spectrum and composition measurements in the expected transition region of the cosmic ray spectrum. Gen2 would also include a veto to enhance the high energy neutrino science. A veto focused on sky coverage would increase the instrumentation footprint of Gen2 to $100 \mathrm{~km}^{2}$, potentially improving the cosmic ray program as well. A veto focused on energy threshold would increase the density of instrumentation, employing 5,000-10,000 $\mathrm{m}^{2}$ of particle detectors to veto air showers down to a few hundred $\mathrm{TeV}$. Such a veto would plausibly produce counts of 50 or more peripheral muons for air showers with $E>30 \mathrm{PeV}$, providing another handle on composition in the region expected for a transition from galactic to extra-galactic cosmic rays.

\section{References}

[1] IceCube Collaboration, A. Achterberg et al., Astropart.Phys. 26 (2006) 155. 
[2] IceCube Collaboration, R. Abbasi et al., arXiv:1207.6326 (2012).

[3] IceCube Collaboration, T. Feusels, K. Rawlins et al., Latest Results on Cosmic Ray Spectrum and Composition from Three Years of IceTop and IceCube, \#334 these proceedings (2015).

[4] IceCube Collaboration, M. G. Aartsen et al., Phys. Rev. D 88, 042004, (2013).

[5] T. Feusels, Ph.D. thesis, University of Gent (2013).

[6] IceCube Collaboration, H. Dembinski, J.G. Gonzalez et al., Surface Muons in IceTop, \#267 these proceedings (2015).

[7] IceCube Collaboration, J.G. Gonzalez et al., Studying Cosmic Ray Composition with IceTop using Muon and Electromagnetic Lateral Distributions, \#338 these proceedings (2015).

[8] A. Haungs, Cosmic Rays from the Knee to the Ankle", Physics Procedia 61 (2015) 425.

[9] IceCube Collaboration, M.G. Aartsen et al., Phys. Rev. Lett. 113, 101101 (2014).

[10] IceCube Collaboration, M.G. Aartsen et al., submitted to Phys. Rev. Lett. (2015).

[11] IceCube Collaboration, M.G. Aartsen et al., arXiv:1412.5106 (2014).

[12] IceCube-Gen2 Collaboration, E. Blaufus, C. Kopper, C. Haack et al., The IceCube-Gen2 High Energy Array, \#1146 these proceedings (2015).

[13] IceCube-Gen2 Collaboration, J. Auffenberg et al., Motivations and Techniques for a Surface Detector to Veto Air Showers for Neutrino Astronomy with IceCube in the Southern Sky, \#1156 these proceedings (2015).

[14] IceCube-Gen2 Collaboration, S. Euler, J.G. Gonzalez et al., Simulation Studies for a Surface Veto Array to Identify Astrophysical Neutrinos at the South Pole, \#1070 these proceedings (2015).

[15] Data compilation from S. Tilav (tilav@udel.edu): (Tibet-III) M. Amenomori et al., Ap. J. 678, 1165 (2008), (ARGO-YBJ) VULCANO Workshop, arXiv:1408.6739v1 (2014), (Kascade) T. Antoni et al., Astropart. Phys. 24, 1 (2005), (Kascade-Grande) Advances in Space Research 53, 10, 1456, (2014), (GAMMA) Samvel Ter-Antonyan, Phy. Rev. D 89, 123003 (2014), (Tunka) V.V. Prosin et al., Nuc. Instr. \& Meth. A756, 94 (2014), (HiRes 2)R.U. Abbasi et al. (HiRes Collaboration), Phys. Rev. Lett. 100 (2008) 101101, T. Abu-Zayyad et al. (TA) Astroparticle Physics 48, 16 (2013), A. Schulz et al. (Auger), Proc. 33rd ICRC, Rio de Janeiro, Brazil (2013), arXiv:1307.5059.

[16] IceCube Collaboration, M. G. Aartsen et al., Phys. Rev. D 87, no. 6, 062002 (2013)

[17] IceCube Collaboration, M. Sutherland et al., Search for High Energy Neutron Point Sources in IceTop, \#250 these proceedings (2015).

[18] IceCube Collaboration, R. Abbasi et al., ApJ 746, 33 (2012).

[19] IceCube Collaboration, M. G. Aartsen et al., ApJ 765, 55 (2013).

[20] IceCube Collaboration, F.T. McNally, P. Desiatti, S. Westerhoff et al., Anisotropy in Cosmic Ray Arrival Directions Using IceCube and IceTop, \#274 these proceedings (2015).

[21] IceCube Collaboration, K. Jero, D. Tosi et al., IceTop as Veto for IceCube, \#1086 these proceedings (2015).

[22] Auger Collaboration, M.C. Medina et al., Nucl. Instrum. Meth. A566 (2006).

[23] IceCube Collaboration, M.G. Aartsen et al., submitted to Astropart. Phys., arXiv:1506.07981 (2015).

[24] Auger Collaboration, A. Aab et al., Phys. Rev. D 90, 012012 (2014). 